\title{
Focus scanning with feedback control for fiber-optic nonlinear endomicroscopy (Conference Presentation) (Withdrawal Notice)
}

Ang Li, Wenxuan Liang, Xingde Li

Ang Li, Wenxuan Liang, Xingde Li, "Focus scanning with feedback control for fiber-optic nonlinear endomicroscopy (Conference Presentation) (Withdrawal Notice)," Proc. SPIE 10040, Endoscopic Microscopy XII, 100400S (1 February 2018); doi: 10.1117/12.2250995

SPIE. Event: SPIE BiOS, 2017, San Francisco, California, United States 


\section{Focus scanning with feedback control for fiber-optic nonlinear endomicroscopy (Conference Presentation) (Withdrawal Notice)}

Proc. SPIE 10040, 100400S (2016)

Online Publication Date: 19 April 2017

Withdrawn from Publication: 1 February 2018

Conference Date: 29-30 January 2017

Conference Location: San Francisco, California, United States

Conference Title: Endoscopic Microscopy XII

Conference Chairs: Guillermo J. Tearney, Thomas D. Wang

Ang Li, Wenxuan Liang, Xingde Li

Johns Hopkins Univ. (United States)

Publisher's Note: This conference presentation, originally published on 19 April 2017, was withdrawn per author request. 